\title{
Productos agrícolas para implementar huertas caseras en Altos de Cazucá, Soacha
}

\section{Agricultural products to implement home gardens in Altos de Cazucá, Soacha}

Fecha de recepción: 3 de marzo de 2018 Fecha de aprobación: 5 de junio de 2018
German Ricardo Paredes-Guzmán ${ }^{1}$

Pedro Antonio Forero-Palomino ${ }^{2}$

DOI: http://doi.org/10.19053/01228420.v15.n2.2018.8394

\section{Resumen}

Altos de Cazucá (Soacha, Cundinamarca) es una zona afectada por el desplazamiento forzado de la población, el cual aumenta los cinturones de miseria del municipio, así como los problemas de malnutrición. Una posible mitigación de estas características podría ser la aplicación de métodos que tengan que ver con la sostenibilidad agrícola, de la cual hace parte la Agricultura Urbana (AU), que contribuye a la producción de especies vegetales que favorecen la soberanía alimentaria y nutricional. Es por ello que es necesario conocer cuáles son los hábitos de consumo, para poder implementar diferentes métodos de establecimiento y producción de AU. Mediante la entrevista semiestructurada a pobladores del barrio Loma Linda de Altos de Cazucá, se lograron identificar los productos agrícolas de mayor consumo en la zona y los más indicados para implementar huertas caseras allí, bajo un modelo de AU. Se concluyó que la arveja y la zanahoria son las de mayor aceptación y mayor consumo, con $90 \%$ y $86 \%$, respectivamente, y que la quinua es el alimento que menos consumen, con un $8 \%$. El mayor consumo de aromáticas y especias es de hierbabuena, sábila y limoncillo, con un porcentaje que varía entre el $58 \%$ y el $48 \%$. Por otra parte, el $26 \%$ de la población encuestada expresó tener pleno conocimientos en temas de agricultura urbana; el $38 \%$, tener conocimientos medianos, y $36 \%$ admitió que no tiene información al respecto; sin embargo, el $98 \%$ está dispuesta a participar en actividades de agricultura urbana.

Palabras clave: agricultura; agricultura urbana; sostenibilidad; vulnerabilidad.

1 M. Sc. Universidad Santo Tomás (Bogotá-Distrito Capital, Colombia). germanparedes@ustadistancia.edu.co. ORCID: 0000-0002-3977-4826.

2 Universidad Santo Tomás (Bogotá-Distrito Capital, Colombia). pedroforero@ustadistancia.edu.co.

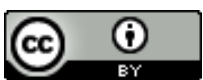




\begin{abstract}
The Loma Linda of Altos de Cazucá neighborhood (Soacha, Cundinamarca) is affected by forced displacement, which increases both the misery belts and the malnutrition problems of the municipality. A potential way to mitigate these issues is the introduction of sustainable agriculture methods such as urban agriculture (UA), which contributes to produce vegetal crops that favor the alimentary and nutritional sovereignty. Knowing the consumption habits of the community is necessary to implement different UA establishment and production methods. Therefore, to identify the main consumed agricultural products in the Loma Linda of Altos de Cazucá neighborhood and select those that can be implemented in a family garden under an UA model, we used a semi-formal interview tool. We found that peas and carrots are the most consumed with $90 \%$ and $86 \%$, respectively, and that quinoa is the least consumed with $8 \%$. Among aromatics and spices, the most consumed are spearmint, aloe, and lemongrass, with percentages that range from 58 to 48 . Additionally, $26 \%$ of the polled population expressed having full and average knowledge in subjects of UA; 38\%, having average knowledge, and 36\% admitted having no information in this regard, however $98 \%$ declared to be willing to participate in UA activities.
\end{abstract}

Keywords: agriculture; sustainability; urban agriculture; vulnerability.

\title{
Para citar este artículo:
}

Paredes-Guzmán GR., Forero-Palomino PA. Productos agrícolas para implementar huertas caseras en Altos de Cazucá, Soacha. Ciencia y Agricultura. 2018; 15(2): 39-45. 


\section{Introducción}

En la coyuntura en que se encuentra Colombia se pueden apreciar varios contrastes, sobre todo, en lo que tienen que ver con el sector económico, los cuales afectan a la población y, por ende, la zona geográfica donde habitan; este tipo de contrastes se pueden apreciar, por ejemplo, en el número de clínicas de cirugía estética en el país, donde más de la mitad de la población vive por debajo de la línea de la pobreza; este tipo de contrastes, posiblemente, se pueden generar por violencia, precarios servicios básicos y falta de un ingreso económico estable de la población, que se encuentra. Es el caso de Altos de Cazucá, zona afectada por el desplazamiento forzado, donde la mayoría de sus habitantes, dadas sus precarias condiciones económicas, se ubican en los suburbios, aumentando los cinturones de miseria de Soacha; problemática que afecta sociocultural, psicológica y físicamente la población (1).

De acuerdo con el estudio "Caracterización de la población vulnerable de la Comuna 4, Altos de Cazucá", realizado en el 2013, se encontró que los ingresos de esta población son inferiores en un $50 \%$ al salario mínimo y que los niveles de indigencia se hacen evidentes en el $20 \%$ de la población. La ACNUR (1) reporta que, entre las enfermedades más comunes en esta zona se encuentran las relacionadas con la desnutrición; de igual forma, Restrepo, Sierra y Bayona (2) mencionan datos sobre actividades laborales, índices de indigencia y número de miembros por familia, entre otros, los cuales permiten dimensionar el problema alimentario.

El difícil acceso a los productos básicos de la canasta familiar ahonda los problemas de malnutrición de la población, a pesar de que Colombia es un país netamente agrícola, este tipo de productos no son asequibles para la población vulnerable, por sus costos. Se plantea como necesario implementar un modelo de producción de agricultura urbana, en el cual participe la comunidad de forma activa, a través de diferentes metodologías, métodos y técnicas.
La seguridad alimentaria y nutricional se ha incluido en Colombia como política nacional; el documento CONPES Social 113 de 2008 la enmarca y define sus orientaciones (3).

La agricultura urbana constituye un modelo fundamental para disminuir de manera creciente los problemas de seguridad alimentaria (4); la participación activa de los facilitadores y la comunidad permite un estrecho vínculo entre el investigador y el productor y la adopción por este de los resultados de las nuevas investigaciones realizadas.

Este estudio, realizado de acuerdo con el documento CONPES Social II, se enmarca en las cuatro dimensiones señalas por este: la disponibilidad, la accesibilidad, el consumo y el aprovechamiento de alimentos que sean producidos por la propia comunidad, ya que el esquema de abastecimiento actual de alimentos en el municipio de Soacha está dado por el sector informal alimentario, que opera como un proceso comercial de alimentos frescos, prelistos o preparados in situ, dentro de un sistema de abastecimiento y distribución de alimentos precario o espontáneo (5).

De igual forma, el PNUD (5) informa que la accesibilidad implica que los alimentos disponibles y los recursos productivos estén al alcance de los hogares y las familias -y de cada uno de sus integrantes- y que no existan restricciones físicas, económicas o culturales para conseguir los alimentos y consumirlos de manera autónoma, en cantidad y calidad suficientes. A su vez, el acceso a los alimentos no puede sacrificar o menguar el goce de otros derechos humanos.

Para identificar los principales productos de consumo, para su posterior implementación de siembra en campo, este estudio se enmarcó bajo las metodologías y técnicas de la Investigación Acción Participativa (IAP), en la cual el docente, los estudiantes y la comunidad participante no son simples espectadores, sino unos trasformadores de su realidad, a través de la producción de un conocimiento propositivo mediante un proceso de debate, reflexión y construcción colectiva de saberes entre los diferentes actores, con el fin de lograr la transformación social. 
En este contexto, se plantea como objetivo identificar los principales productos agrícolas para implementar huertas caseras, bajo un modelo de agricultura urbana.

\section{Materiales y métodos}

Altos de Cazucá se encuentra localizado en las coordenadas $0.24^{\prime} 35^{\circ} 4^{\prime \prime}$ latitud norte y $74^{\circ} 11^{\prime} 4.088^{\prime \prime}$ longitud oeste; comprende la loma de Sierra Morena y el embalse de Terreros, y presenta la mayor zona de conurbación con Bogotá. A partir de estimativos, se conoce que la zona de Altos de Cazucá comprende 30 barrios, ubicados en dos franjas: una localizada al costado occidental de la loma Sierra Morena y que colinda con la Autopista Sur (franja 1); allí se encuentran los barrios Balcanes, La capilla, Casa Loma, Loma Linda, Minuto de Dios, Carlos Pizarro, Julio Rincón, Villa Mercedes, Luis Carlos Galán I, Luis Carlos Galán II, Santo Domingo y Arrayanes, y la otra, localizada en la ladera frente al embalse de Terreros y la Ciudadela Sucre (franja 2).

Por las condiciones que presenta la zona, se realizaron 100 entrevistas, con el Método de Caso Social Individual planteado por Guzmán (6), el cual incorpora las técnicas de observación y entrevista, las cuales se emplearon para recabar datos sobre la situación individual, con miras a elaborar un diagnóstico que sirva de apoyo al tratamiento social. La observación se emplea para estudiar las características del medioambiente de la comunidad y las relaciones que esta manifiesta frente a un problema, y la entrevista cumple un papel importante desde varios puntos de vista:

a. Sirve como instrumento para establecer una relación profesional adecuada.

b. Permite recoger sistemáticamente información específica sobre la comunidad, como datos personales, familiares, sobre su problema y otros de importancia.

Las variables de análisis fueron: consumo de alimentos (V1), preferencias de consumo (V2), consumo de aromáticas y especias (V3), frecuencia de uso de aromáticas y especias (V4), conocimiento sobre agricultura urbana (V5), interés por producir bajo la metodología de agricultura urbana (V6) y complicaciones para producir bajo el modelo de agricultura urbana (V7).

Para realizar el análisis de las variables se utilizó el programa Microsoft ${ }^{\oplus}$ Excel $^{\oplus}$; los datos obtenidos de las encuestas se introdujeron para graficar los resultados y realizar el análisis correspondiente.

\section{Resultados y discusión}

La Política Pública de Seguridad Alimentaria y Nutricional se estableció en el año 2007, mediante el Decreto 508; en ella se define el derecho a la alimentación como el "derecho a tener disponibilidad y acceso en forma regular, permanente y libre a una alimentación adecuada y suficiente, que responda a las tradiciones culturales de la población, así como a agua suficiente, salubre y aceptable para el uso personal y doméstico, que garanticen una vida, individual y colectiva, satisfactoria y digna"; sin embargo, en Altos de Cazucá no se refleja, como lo confirma el informe final del Programa Mundial de Alimentos sobre la evaluación de la seguridad alimentaria y nutricional de las poblaciones vulnerables de Colombia, del 2011; este informe reporta que el $11.5 \%$ de los hogares tienen un consumo inadecuado (límite o pobre), aumentando la cifra en comparación con la encuesta de seguimiento realizada en el 2010, donde esta proporción alcanzaba el $9.7 \%$.

La proporción de desplazados inscritos en el Registro Único de Población Desplazada (RUPD) con al menos un indicador de insuficiencia alimentaria alcanza el $65.2 \%$, y la de los no inscritos, el 65.9 $\%$.

Igualmente, la presencia simultánea de síntomas de alimentación insuficiente fue alrededor del 37 $\%$ en las personas inscritas y no inscritas.

El mismo estudio mostró que las personas desplazadas recientemente tienen más dificultades para acceder a los alimentos. Este grupo, al no tener acceso a tierras para cultivar, pasa a depender altamente del mercado para obtener sus alimentos, de modo que la principal limitante es la falta de 
ingresos estables y suficientes; por ello, estas familias recurren con mayor frecuencia a la compra al crédito (fiado).

La FAO (7) define la disponibilidad de alimentos como la oferta de suficientes alimentos en los mercados locales, en cantidad y calidad adecuadas, obtenida a través de la producción nacional y las importaciones. Según los datos obtenidos en la entrevista realizada en el barrio Loma Linda, el consumo de verduras, hortalizas y leguminosas es del $56 \%$; el consumo de alimentos de este grupo es muy benéfico para la salud, puesto que proporciona nutrientes esenciales como el hierro y las proteínas. Le sigue el consumo de frutas, con un $25 \%$; la baja ingesta de frutas disminuye, principalmente, el aporte de vitamina C, carotenos y fibra. Las aromáticas son el producto que menos consumen (19\%).

Las verduras, hortalizas y leguminosas que mayor aceptación tienen en la comunidad, por su palatabilidad, son las arvejas y las zanahorias, con un 90 $\%$ y $86 \%$, respectivamente; según el Minsalud-ENSIN (9), la arveja y la zanahoria son los alimentos de mayor consumo y cantidad promedio/persona/ día; la arveja tiene un $16.9 \%$ de individuos que la consumen, y la zanahoria, un 28,7 \% (Figura 1).

Le sigue un grupo de productos en los que se encuentran el tomate, la cebolla, el frijol, la lechuga, las acelgas, las espinacas, la remolacha y el calabacín, en un rango de porcentaje de 78 a 60 (Figura 1); dentro del estudio de ENSIN solo mencionan el tomate, la cebolla y el frijol: $38.7 \%, 30.1 \%$ y 15.0 $\%$, respectivamente, de individuos que los consumen; este es el reflejo del consumo nacional en Altos de Cazucá, en los productos referenciados; los demás productos en estudio no se encuentran referenciados.

Los productos que se encuentran en un rango medio de consumos son ahuyama, repollo y coliflor, los cuales no superan el $60 \%$. En el caso del repollo, solo el $6.7 \%$ de los individuos reportan su consumo, situándolo en un rango bajo. Por otra parte, los productos de menor aceptación para su consumo son las coles y los espárragos, con un porcentaje de $24 \%$ y $20 \%$, respectivamente, y la quinua y los puerros son los productos con el menor agrado entre los pobladores de la comunidad encuestada, solo cuentan con el $8 \%$ de aceptación (Figura 1); cabe anotar que la quinua, que se encuentra en el rango de menor agrado, no la ha probado, en ninguna presentación, la mayoría de comunidad (el $86 \%$ ).

El consumo se relaciona con las condiciones socioeconómicas de la población, es así como varía de acuerdo con el nivel de Sisben: $36.7 \%, 66.5 \%$ y $56.5 \%$ de personas que consumen leguminosas, verduras y frutas, respectivamente, en el Sisben I (9).

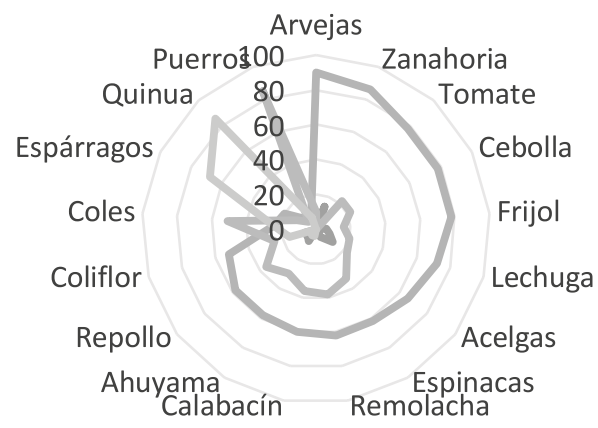

$\longrightarrow$ LE GUSTA

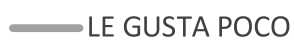

NO LE GUSTA NO LA HE PROBADO

Fig. 1. Preferencia de consumo.

Las especias y aromáticas, aunque son las de menos consumo por la comunidad, son una buena opción para ser producidas en huertas caseras, por sus beneficios y su ciclo corto de producción; el mayor consumo se presenta entre las especies de hierbabuena, sábila y limoncillo, con un porcentaje que varía entre el $58 \%$ y el $48 \%$, seguido por el perejil, la caléndula, la menta, el poleo, el romero, el paico, la ruda y el toronjil, con un menor consumo con respecto al primer grupo de consumo, con un rango del $42 \%$ al $30 \%$. El consumo de estas 
especies en particular puede ser para dolencias (69 $\%$ ) o habitual en infusiones (9\%); entre las especies con un consumo moderado se encuentran a la mejorana, la albahaca de la virgen, el cidrón, la yerbamora y la archucha pepino, productos que no superan el $22 \%$, según lo reportado por la comunidad; estos datos pueden ser para el consumo moderado, con una frecuencia de 15 días, reportado por el $10 \%$ de la población encuestada.

Entre las aromáticas con menor consumo, con porcentajes que varían entre el $8 \%$ y el $2 \%$, están el cimarrón, el orégano francés, la mirra, la verdolaga blanca, la achira-capacho, la sanguinaria y la lengua de vaca, posiblemente por el desconocimiento de sus beneficios y por el bajo consumo de aromáticas en general: se reporta que el $4 \%$ de la comunidad solo consume aromáticas una vez al mes (Figura 2).

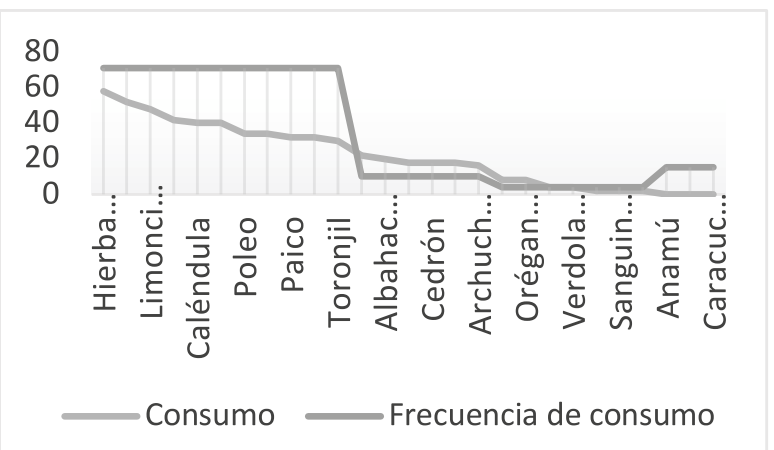

Fig. 2. Preferencia de consumo vs. frecuencia de consumo.

Este consumo es corroborado por Cardona y Barrientos en el 2007, quienes afirman que el uso tradicional de las plantas aromáticas se fundamenta en cultivar las especies más comerciales en pequeñas parcelas o jardines como algo marginal, y recolectar las especies no cultivadas y menos comerciales. Sus usos son principalmente medicinales, pero también se destinan a su consumo como condimentos, usos esotéricos, infusiones e insecticidas; de igual forma, en el mismo estudio, más de la mitad de los encuestados comercializa especies aromáticas para, principalmente, consumo en fresco.
El $13 \%$ de la comunidad reporta que no conocen y que no consumen aromáticas en ninguna de sus formas ( $2 \%)$. Aromáticas conocidas como anamú, caracola y caracucho, no son consumidas por la comunidad, según los resultados obtenidos.

En cuanto al conocimiento de la población sobre temas de agricultura urbana (V5), la entrevista reporta que existe un pleno y un mediano conocimiento, con un $26 \%$ y $38 \%$, respectivamente, frente a un $36 \%$ de la población que no tienen el suficiente conocimiento del tema.

Sin embargo, el $98 \%$ de la población está dispuesta a participar en actividades que tengan que ver con la agricultura urbana (V6), aunque a los pobladores del barrio Loma Linda les preocupa la logística para adelantar proceso de agricultura urbana, sobre todo en lo que tiene que ver con el espacio de producción (52\%), los implementos o herramientas (12\%) y los recueros económicos $(11 \%)$, por el grado de complicación. Otros factores que les preocupan son el clima y la fertilización para las plantas, que junto con el poco conocimiento son las variables menos preocupantes para producir bajo condiciones de agricultura urbana, con porcentajes que varían entre el $9 \%$ y el $3 \%$ (Figura 3).

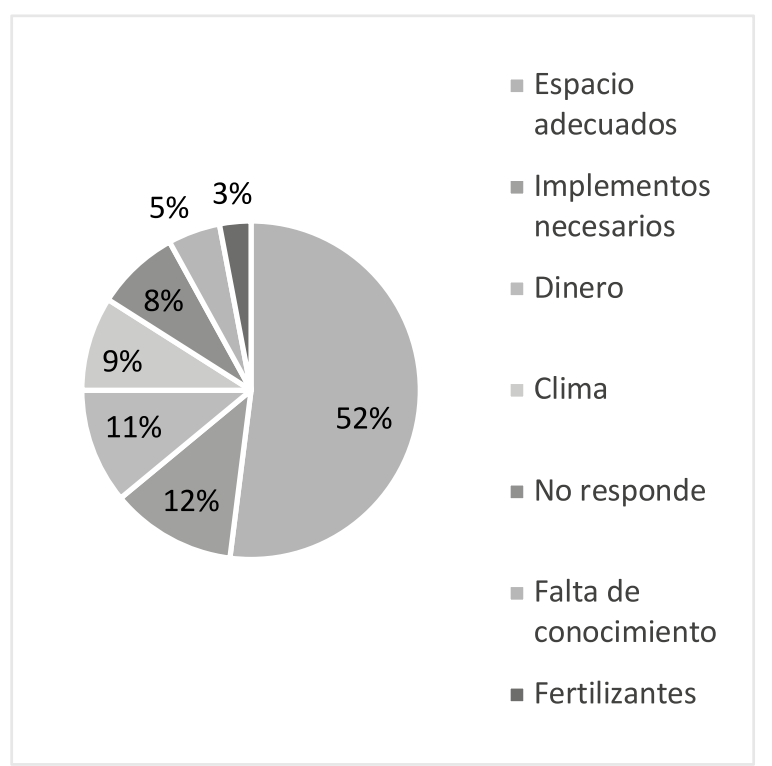

Fig. 3. Principales complicaciones para producir bajo el modelo de agricultura urbana. 
De acuerdo con Cantor (11), la agricultura urbana emplea recursos locales humanos y físicos, y sus productos se destinan para el autoconsumo o para la distribución en el mercado. Los puntos de discusión entre las definiciones del concepto son: a) la amplitud de la actividad, es decir, si solamente produce alimentos o si genera otros productos; b) la marginalidad espacial de los productores, es decir, si los productores se encuentran únicamente en zonas deprimidas; c) si la actividad reutiliza, necesariamente, recursos físicos, aun siendo escasos, y d) si exclusivamente emplea pequeñas áreas.

Por otra parte, como lo mencionan Marulanda (12) y Canidad y Murciano (13), citados por Cantor (11), la agricultura urbana es un medio que reactiva ocupaciones que validan socialmente a personas con discapacidad, a hombres con edades avanzadas y, principalmente, a mujeres; de igual manera, la huerta tiene un valor simbólico para las personas, ya que la actividad agropecuaria en la ciudad incorpora cierto valor al paisaje y representa valoraciones importantes en la vida cotidiana de los agricultores.

\section{Conclusiones}

En el barrio Loma Linda, el acceso a los alimentos depende altamente de los mercados, y las limitaciones de la comunidad para su consumo es, principalmente, la falta de ingresos. La quinua es un alimento que la comunidad no ha probado en ninguna presentación $(86 \%)$, debido, posiblemente, a la poca difusión de sus atributos como alimento y de su forma de preparación. La hierbabuena, la sábila y el limoncillo, con un porcentaje que varía entre el $58 \%$ y el $48 \%$, son las aromáticas y especias de mayor consumo entre la comunidad encuestada, que en su mayoría las utilizan para dolencias $(69 \%)$ y consumo habitual en infusiones $(9 \%)$; esto es confirmado por Cardona y Barrientos (2007), quienes afirman que sus usos son principalmente para medicina, pero también se destinan a su consumo como condimentos, usos esotéricos, infusiones e insecticidas.

El $26 \%$ de la población encuestada del barrio Loma Linda afirma que tiene un conocimiento pleno en temas relacionados con la agricultura urbana; el 38
$\%$, un conocimiento mediano, y el $36 \%$, que no tiene información al respecto; sin embargo, el 9 $8 \%$ está dispuesta a participar en actividades relacionadas con producción en agricultura urbana. Esto se relaciona con lo que afirman varios autores en cuanto a que la agricultura urbana es un medio que reactiva ocupaciones que validan socialmente a personas con discapacidad, hombres con edades avanzadas $\mathrm{y}$, principalmente, a mujeres.

\section{Referencias}

(1) ACNUR. Altos de Cazucá, Hasta cuándo en el olvido. 2003. Disponible en: http://www.ACNUR.org/t3/uploads/media/COI_1582. pdf?view $=1$.

(2) Restrepo de Peña BR., Sierra Borrás JE., Bayona Cabeza M. La Empresa de Economía Solidaria, una alternativa de solución para comunidades vulnerables en el municipio de Soacha, Comuna IV, Altos de Cazucá. Revista Arbitrada del Centro de Investigación y Estudios Gerenciales, 2014; 4: 144-159.

(3) Minagricultura. Documento CONPES 113 - Política nacional de seguridad alimentaria y nutricional (PSAN). 2008. Disponible en: https://www.minagricultura.gov.co/Normatividad/Conpes/ conpes_113_08.pdf.

(4) Hernández L. La agricultura urbana y Caracterización de sus sistemas productivos y sociales, como vía para la seguridad alimentaria en nuestras ciudades. Cultivos Tropicales. 2006; 27 (2): 13-25.

(5) PNUD. Estado de avance de los Objetivos de Desarrollo del Milenio. 2012. Disponible en: http://www.pnud.org.co/2012/ odm2012/odm_soacha.pdf.

(6) Guzmán, L. Metodología y técnicas de investigación en el Trabajo Social. 1985. Disponible en: http://www.ts.ucr.ac.cr/binarios/docente/pd-000194.pdf.

(7) Food and Agriculture Organization -FAO-. Declaración de Roma sobre la Seguridad Alimentaria Mundial. 1996. Disponible en: http://www.fao.org/docrep/003/w3613s/w3613s00.htm.

(8) Programa Mundial de Alimentos. Evaluación de la seguridad alimentaria y nutricional en las poblaciones vulnerables de Colombia. 2011. Disponible en: http://documents.wfp.org/stellent/ groups/public/documents/ena/wfp240719.pdf.

(9) MINSALUD. Encuesta nacional de la situación nutricional en Colombia. 2005. Disponible en: https://www.minsalud.gov.co/sites/ rid/Lists/BibliotecaDigital/RIDE/VS/ED/GCFI/Ensin\%202005.pdf.

(10) Cardona J., Barrientos J. Producción, uso y comercialización de especies aromáticas en la región Sumapaz, Cundinamarca. Revista Colombiana de Ciencias Hortícolas. 2011; 5(1): 114-129. DOI: https://doi.org/10.17584/rcch.2011v5i1.1258.

(11) Cantor K. Agricultura urbana: sostenibilidad y medios de vida - Experiencias en Ciudad Bolívar, Altos de Cazucá y Ciudadela Sucre. Tesis de maestría. Universidad Javeriana. 2009.

(12) Marulanda C. Microhuertas familiares: cultivo de esperanzas con rendimientos de paz. 2007.

(13) Canidad M., Murciano Y. Notes on a Cuban experience: The duality of equality. Revista WE International. 1998; 44(45): 14-17. 
